\title{
Dengzhan shengmai capsule combined with donepezil hydrochloride in the treatment of Alzheimer's disease: preliminary findings, randomized and controlled clinical trial
}

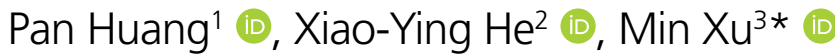

\begin{abstract}
SUMMARY
OBJECTIVE: To observe the effects of Dengzhan Shengmai capsule combined with donepezil hydrochloride on cognitive function, daily living ability, and safety in patients with Alzheimer's disease.

METHODS: A total of 294 patients with Alzheimer's disease were randomly divided into a treatment group and a control group, 147 cases each group. The control group was given oral donepezil hydrochloride $5 \mathrm{mg}$ once a day, and the treatment group was given oral Dengzhan Shengmai capsule $0.36 \mathrm{~g}$ three times a day, based on the control group.

RESULTS: At 3 and 6 months of treatment, the ADAS-cog score of the treatment group was $48.69 \pm 6.23$ and $44.24 \pm 5.53$; for the control group, $45.48 \pm 5.94$ and $41.57 \pm 5.10$. The difference between the two groups is statistically significant $(p<0.05)$. At 3 and 6 months of treatment, the NO level in the treatment group was $(46.28 \pm 6.68) \mathrm{umol} / \mathrm{I},(43.55 \pm 7.92) \mathrm{umol} / \mathrm{l}$, and the control group was (42.95 \pm 7.92$)$ umol/I, (38.89 \pm 5.93$)$ umol/I. The differences between both groups were statistically significant $(p<0.05)$. At 3 and 6 months of treatment,

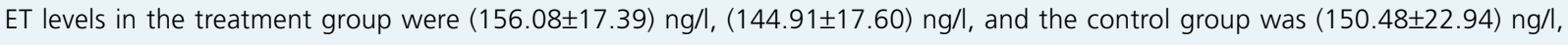
$(135.04 \pm 10.08) \mathrm{ng} / \mathrm{l}$. Correlation analysis showed that ADAS-cog score was negatively correlated with NO and ET ( $<<0.001)$.

CONCLUSIONS: Dengzhan Shengmai capsule combined with donepezil hydrochloride can improve cognitive function and the living capacity of patients with Alzheimer's disease, reduce the production of neurotoxic substances NO and ET, and provide higher safety. KEYWORDS: Alzheimer's disease. Donepezil. Flavonoids. Medicine, traditional Chinese.
\end{abstract}

\section{INTRODUCTION}

Alzheimer's disease (AD) is a neurodegenerative condition of the central nervous system (CNS) characterized by progressive cognitive and behavioral impairment in older adults and preseniles ${ }^{1}$. In China, the incidence of $\mathrm{AD}$ in people over 65 years of age is $3-7 \%$, resulting in a total of $6-8$ million $\mathrm{AD}$ patients in China ${ }^{2}$. Recently, studies have shown that oxidative stress, endothelial cell damage, and mitochondrial dysfunction are involved in the pathogenesis of AD. Donepezil hydrochloride is currently the only drug supported by clinical evidence for being effective in the treatment of AD. Dengzhan Shengmai Capsule is a traditional Chinese medicine that contains flavonoids, which regulate vascular endothelial cells and protect mitochondria. Recently, the efficacy of Dengzhan Shengmai Capsule combined with donepezil hydrochloride in the treatment of $\mathrm{AD}$ has been confirmed in our department.

\footnotetext{
'People1s Hospital of DeYang City, Department of Neurology - DeYang, China.

${ }^{2}$ The Affiliated Hospital of Southwest Medical University, Department of Neurology - Luzhou, China.

${ }^{3}$ The second People's Hospital of DeYang City, Department of Neurology - DeYang, China

*Corresponding author: 782067723@qq.com

Conflicts of interest: the authors declare there is no conflict of interests. Funding: none.

Received on October 25, 2020. Accepted on November 16, 2020.
} 


\section{METHODS}

\section{Clinical information}

A total of 294 patients with $\mathrm{AD}$ who were treated in the neurology clinics of our hospital or as inpatients, from July 2015 to November 2017, were selected for the study. Patients were randomly assigned to either the treatment or control groups. The treatment group consisted of 147 cases, of which 26 were male and 23 were female. Patients were aged 67-84, with an average age of $70.93 \pm 5.47$ years. The control group consisted of 25 males and 24 females, aged between 66-81, with an average age of $70.48 \pm 6.56$ years.

The inclusion criteria for the study were patients who meet the diagnostic criteria for $\mathrm{AD}^{3}$; patients $>60$ years of age; patients who could complete the study; and patients with brain CT or MRI who did not show signs of cerebral hemorrhage, cerebral infarction, and intracranial lesions. The exclusion criteria for the study were patients with other types of dementia, such as vascular dementia; patients with advanced Parkinson's disease; patients with intracranial infections, schizophrenia, or depression; patients with severe heart, liver, lung, and kidney dysfunction; patients previously treated for dementia; and patients allergic to Dengzhan Shengmai Capsule or donepezil hydrochloride.

The study was approved by the hospital ethics committee and all subjects were recruited under written informed consent. The Ethical Approval Number is 2012-10-003. No significant difference in the general patient characteristics was observed between both groups ( $p>0.05$ ), as summarized in Table 1 .

Table 1. Comparison of clinical data in two groups.

\begin{tabular}{|c|c|c|}
\hline Item & $\begin{array}{c}\text { Treatment } \\
(n=147)\end{array}$ & $\begin{array}{l}\text { Control } \\
(n=147)\end{array}$ \\
\hline Age (year) & $69.12 \pm 5.28$ & $69.31 \pm 5.41$ * \\
\hline $\operatorname{Sex}(M / F)$ & $75 / 72$ & $74 / 73^{*}$ \\
\hline HR (Time/min) & $78.52 \pm 15.18$ & $71.48 \pm 12.30 *$ \\
\hline Weight (kg) & $64.28 \pm 6.78$ & $65.40 \pm 7.88^{*}$ \\
\hline $\mathrm{BMI}\left(\mathrm{kg} / \mathrm{m}^{2}\right)$ & $22.40 \pm 1.92$ & $22.89 \pm 2.06^{*}$ \\
\hline $\mathrm{SBP}(\mathrm{mmHg})$ & $120.71 \pm 14.99$ & $119.14 \pm 14.71 *$ \\
\hline $\mathrm{DBP}(\mathrm{mmHg})$ & $74.42 \pm 10.51$ & $73.57 \pm 12.25^{*}$ \\
\hline Education (year) & $7.14 \pm 2.38$ & $7.10 \pm 2.28 *$ \\
\hline \multicolumn{3}{|c|}{ Combined disease $(n \%)$} \\
\hline Hyperlipidemia & $23(15.64)$ & $25(17.00)^{*}$ \\
\hline Hypertension & $17(11.56)$ & $16(9.41)^{*}$ \\
\hline Diabetes & $10(6.80)$ & $11(7.48)^{*}$ \\
\hline
\end{tabular}

HR: heart rate; BMI: body mass index; SBP: systolic blood pressure; DBP: diastolic blood pressure. Compared to the treatment group, * $p>0.05$.

\section{Treatment method}

Patients in the control group received $5 \mathrm{mg}$ of donepezil hydrochloride orally once a day (specification $5 \mathrm{mg}$ /piece, Eisai (China) Pharmaceutical Co., Ltd., approval number: Sinopharm standard H20050978). The treatment group was based on the control group, who received $0.36 \mathrm{~g}$ Dengzhan Shengmai Capsule three times a day (specification: $0.18 \mathrm{~g} /$ capsule, Yunnan Biological Valley Dengzhanhua Pharmaceutical Co., Ltd., approval number: Sinopharm standard word H20054470). Patients in both groups were treated over six months.

\section{Observation indicators}

Cognitive function was measured with cognitive function scoring. A daily living capacity score and the adverse drug reactions of the two groups of patients before treatment and at three and six months of treatment were recorded. Cognitive function scores were assessed using the ADAS-cog scale, including structure, language, immediate recall and recognition of words, orientation, and the use of ideas. The total possible score was 70 points, in which a higher score indicated worse cognitive function. The daily living capacity score was assessed using the AD Collaborative Research Daily Ability Scale (ADCS-ADL) that consists of 19 ADCS-ADL scales with a total score of 54 points. A higher score indicated a better daily living capacity.

Fasting peripheral venous blood was collected in the early morning before treatment, three months and six months after treatment; the serum levels of nitric oxide and endothelin were measured using enzyme-linked immunosorbent assay after serum isolation. Nitric oxide (NO) detection kit was provided by the American Adlitteram Diagnostic Laboratorues Company (batch number: X01872); endothelin (ET) detection kit was provided by Shanghai Hengyuan Biotechnology Company (batch number: 20150219).

\section{Statistical analysis}

Statistical analysis was performed using SPSS17.0 software. Measurement data were expressed as mean \pm standard deviation $(x \pm s)$, comparison between groups was tested by $\mathrm{t}$ test; count data were compared by percentage (\%), and comparison between groups was tested by chi-square $\left(\chi^{2}\right)$ test. $\mathrm{p}<0.05$ indicates a statistically significant difference.

\section{RESULTS}

\section{Clinical data}

All participants from the treatment and control groups completed the study and were included in the analysis. The demographic characteristics, comorbid diseases, and laboratory tests 
are shown in Table 1 . All variables were not significantly different between the two groups. ( $p>0.05$ ). There was no significant difference in ADAS-cog, ADCS-ADL scores between the two groups of patients before treatment $(\mathrm{p}>0.05)$. The scores of ADAS-cog and ADCS-ADL in the treatment group at three months and six months after treatment were significantly different from those in the control group $(\mathrm{p}<0.05)$ (Table 2). There was no significant difference in the levels of $\mathrm{NO}$ and $\mathrm{ET}$ between the two groups of patients before treatment $(\mathrm{p}>0.05)$.
The differences between the treatment group and the control group at three and six months after treatment were statistically significant $(\mathrm{p}<0.05)$ (Table 3$)$. The Spearman correlation method was used to analyze the correlation between ADAS$\operatorname{cog}$ scores, and NO and ET levels in the treatment group. The results showed that ADAS-cog scores were significantly negatively correlated with $\mathrm{NO}$ and ET levels $(\mathrm{r}=-0.887,-0.608$, $\mathrm{p}<0.05)$. During the six months of treatment, adverse drug reactions in the treatment group were as follows: three cases

Table 2. Comparison of Alzheimer's disease assessment scale-cognitive scores and Alzheimer's disease cooperative study activities of daily living between the two groups $(x \pm s)$.

\begin{tabular}{l|c|c|c|c|c|c}
\multirow{2}{*}{ Item } & \multicolumn{3}{|c|}{ Treatment $(\mathrm{n}=147)$} & \multicolumn{3}{c}{ Control $(\mathrm{n}=147)$} \\
\cline { 2 - 7 } & $\begin{array}{c}\text { Before } \\
\text { treatment }\end{array}$ & 3 months & 6 months & $\begin{array}{c}\text { Before } \\
\text { treatment }\end{array}$ & 3 months & 6 months \\
\hline ADAS-cog & $51.17 \pm 8.67$ & $45.48 \pm 5.94$ & $41.57 \pm 5.10$ & $52.04 \pm 9.16^{*}$ & $48.69 \pm 6.23 \#$ & $44.24 \pm 5.53 \#$ \\
\hline ADCS-ADL & $39.02 \pm 5.28$ & $43.91 \pm 4.25$ & $46.57 \pm 3.86$ & $38.89 \pm 5.13^{*}$ & $40.77 \pm 4.44 \#$ & $42.46 \pm 5.18 \#$ \\
\hline
\end{tabular}

ADAS-cog: Alzheimer's disease assessment scale-cognitive; ADCS-ADL: Alzheimer's disease cooperative study - activities of daily living. Compared to the treatment group, ${ }^{*} p>0.05, \# p<0.05$

Table 3. Comparison of no and et levels between two groups( $x \pm s)$.

\begin{tabular}{c|c|c|c|c|c|c}
\multirow{2}{*}{ Item } & \multicolumn{3}{|c|}{ Treatment $(\mathrm{n}=147)$} & \multicolumn{3}{c}{ Control $(\mathrm{n}=147)$} \\
\cline { 2 - 7 } & $\begin{array}{c}\text { Before } \\
\text { treatment }\end{array}$ & 3 months & 6 months & $\begin{array}{c}\text { Before } \\
\text { treatment }\end{array}$ & 3 months & 6 months \\
\hline NO $(\mathrm{umol} / \mathrm{l})$ & $49.25 \pm 10.97$ & $42.95 \pm 7.92$ & $38.89 \pm 5.93$ & $49.17 \pm 9.18^{*}$ & $46.28 \pm 6.68 \#$ & $43.55 \pm 7.92 \#$ \\
\hline ET $(\mathrm{ng} / \mathrm{l})$ & $158.59 \pm 24.13$ & $150.48 \pm 22.94$ & $135.04 \pm 10.08$ & $159.95 \pm 20.16^{*}$ & $156.08 \pm 17.39 \#$ & $144.91 \pm 17.60 \#$ \\
\hline
\end{tabular}

Compared to the control group, * $p>0.05, \# p<0.05$.

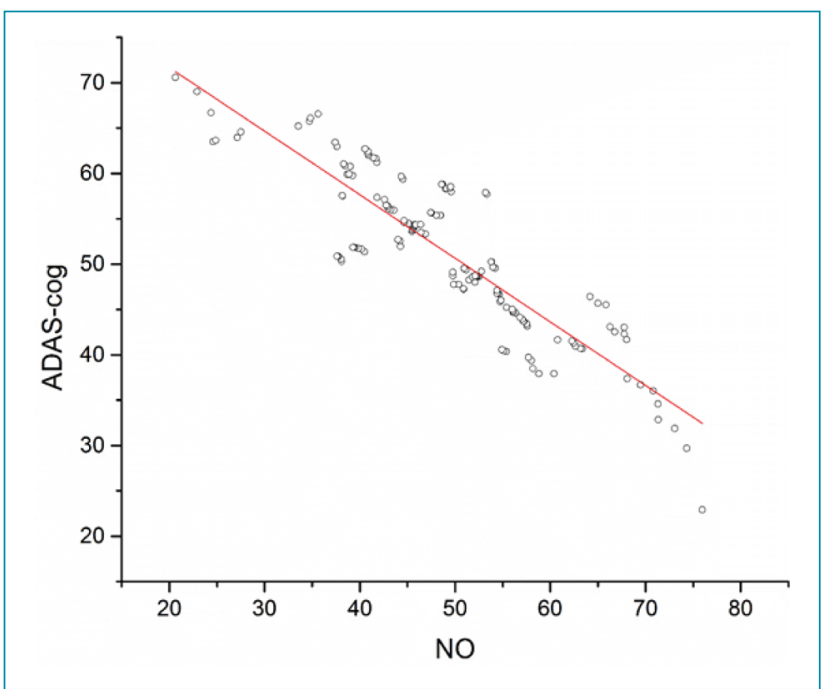

Figure 1. Correlation analysis between Alzheimer's disease assessment scale-cognitive with Nitric oxide.

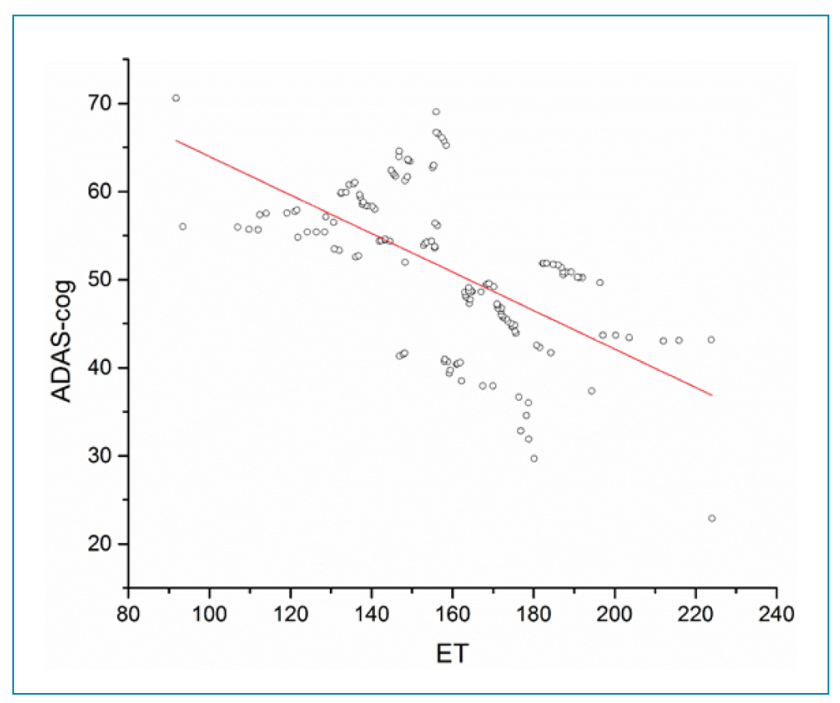

Figure 2. Correlation analysis between Alzheimer's disease assessment scale-cognitive with Endothelin. 
of nausea, two cases of insomnia, one case of headache, two cases of dizziness, one case of diarrhea, one case of rash, and dynamic monitoring of liver and kidney function, and electrocardiogram during the whole course showed no significant dynamic changes. Adverse drug reactions in the control group were as follows: two cases of nausea, three cases of insomnia, two cases of rash, one case of fatigue, and dynamic monitoring of liver and kidney function, and electrocardiogram during the whole course showed no significant dynamic changes. The incidence of adverse reactions in the treatment group and the control group was 6.81 and $5.44 \%$, and the difference was not statistically significant $(\mathrm{p}>0.05)$.

\section{DISCUSSION}

$\mathrm{AD}$ is one of the most common chronic diseases in older people and is a major global public health concern. Since ancient times, traditional Chinese medicine has provided a detailed description of the disease. "Qianjin Yaofang" refers to the disease as a person with more than 50 years of age and dysfunction. "Laziness" and "Medical Forest Corrects Errors" describes the fact that "in children with no memory, the brain is not full, and in those with advanced memory, the brain gradually declines".

Traditional Chinese medicine believes that the pathogenesis of $\mathrm{AD}$ involves "the lack of marrow sea and the use of a magic machine". Its pathological properties have been described as "the time when the essence is true, the essence is yin essence, qi and blood deficiency, and the essence is Qi, fire, Sputum and stasis are blocked in the brain". Therefore, "empty marrow sea, liver and kidney deficiency, heart and liver fire, qi stagnation and blood stasis, phlegm and turbidity" are the root causes of this disease ${ }^{4}$.

For the treatment of $\mathrm{AD}$, the principles of traditional Chinese medicine are Kaiyuzhutan, Huoxuetongqiao, Pingganxiuehuo treatment of its symptoms, tonicity, righteousness, and filling the brain. At present, the treatment of AD uses drugs. Donepezil hydrochloride in Western medicine can reversibly inhibit the hydrolysis of acetylcholine by acetylcholinesterase and can be used in the treatment of mild, moderate, and severe $\mathrm{AD}^{5,6}$. However, Western medicine often has more toxic side-effects compared to traditional Chinese medicine.

Dengzhan Shengmai Capsule is a traditional Chinese medicine preparation developed from several precious medicinal materials, such as Dengzhan Asarum, Ginseng, Schisandra, and Ophiopogon. The main component of the drug is Erigeron breviscapus, which is a flavonoid compound extracted from Erigeron breviscapus ${ }^{7}$. Schisandrol contained in Schisandra chinensis has been shown to have a protective effect on synapses and mitochondria. Also, basic research has shown that Schisandrolone can significantly improve the learning ability of $\mathrm{AD}$ model rats ${ }^{8}$.

In this study, through the results of a randomized controlled clinical trial, we found that donepezil hydrochloride combined with the Dengzhan Shengmai Capsule significantly improved ADAS-cog, ADCS-ADL scores in patients with $\mathrm{AD}$ compared to donepezil hydrochloride alone.

There are currently many hypotheses concerning the pathogenesis of $\mathrm{AD}$ in Western medicine. The $\beta$-amyloid waterfall hypothesis suggests that imbalance in the production and clearance of $A \beta$ is the initial event leading to neuronal degeneration and dementia'. The tau protein theory believes that over-phosphorylation of the tau protein affects the stability of neuron skeleton tubulin leading to the formation of neuron fiber tangles that disrupt the normal functions of neurons and synapses ${ }^{10}$. These two theories have been confirmed based on the histopathological manifestations of $\mathrm{AD}$, which include neuritic and neuro-inflammatory plaques formed by beta-amyloid deposition, and neurofibrillary tangles formed by hyperphosphorylated tau protein.

Recently, oxidative stress has been implicated in the onset of $\mathrm{AD}^{11}$. Oxidative stress refers to the imbalance between the production and removal of oxygen free radicals in the body, resulting in the accumulation of reactive oxygen species (ROS) and reactive nitrogen species (RNS). Nitric oxide (NO) is one of the common species in the RNS family. Studies have shown that NO can regulate the synaptic plasticity of neurons and that excessive increases in $\mathrm{NO}$ can directly damage neuronal cells ${ }^{12}$. NO can also activate multiple intracellular signals to enhance the activity of P53, resulting in increased expression of pro-apoptotic caspases to promote neuronal cell apoptosis ${ }^{13}$.

In this study, we showed that the expression of $\mathrm{NO}$ in the peripheral serum of patients with $\mathrm{AD}$ significantly increased. Correlation analysis showed that $\mathrm{NO}$ was negatively correlated with the ADS-cog score, which confirmed that $\mathrm{NO}$ is involved in the pathogenesis of $\mathrm{AD}^{10,14,15}$. Brain tissue hypoperfusion and microvascular endothelial changes have also been linked to the pathogenesis of $\mathrm{AD}^{16,17}$, Endothelin-1 (ET-1) is currently the strongest known vasoconstrictor. A large amount of ET-1 production can cause intracranial vasoconstriction, resulting in chronic cerebral blood flow insufficiency, thus causing vascular and mitochondrial dysfunction, and increased production of ROS, leading to $\mathrm{AD}^{17,18}$.

We showed that the level of ET-1 in AD patients was significantly higher than that in the normal population. Correlation analysis showed that ET-1 was negatively correlated with the ADAS-cog score, suggesting that ET-1 is involved in the pathogenesis of AD. Moreover, the levels of NO and ET-1 in the 
experimental group were significantly lower than in the control group, in March and June, after treatment. These data suggest that Dengzhan Shengmai Capsules can reduce ET-1 by relaxing intracranial blood vessels in AD patients. The release of ET-1 can also protect mitochondria by reducing the production of the oxidative stress product NO.

\section{CONCLUSIONS}

Dengzhan Shengmai Capsule combined with donepezil hydrochloride can be used to effectively treat $\mathrm{AD}$ by significantly improving the ADAS-cog scores of AD patients. Reduced levels of neurotoxic substances (NO and ET) were also observed; patients showed an improved quality of life when compared to $\mathrm{AD}$ patients without adverse drug reactions.

\section{AUTHORS" CONTRIBUTIONS}

PH: Formal Analysis, Writing - Original Draft, Writing Review \& Editing. XH: Formal Analysis, Writing - Original Draft, Writing - Review \& Editing. MX: Formal Analysis, Writing - Original Draft, Writing - Review \& Editing.

\section{REFERENCES}

1. Nobili F, Arbizu J, Bouwman F, Drzezga A, Agosta F, Nestor P, et al. European Association of Nuclear Medicine and European Academy of Neurology recommendations for the use of brain 18 F-fluorodeoxyglucose positron emission tomography in neurodegenerative cognitive impairment and dementia: Delphi consensus. Eur J Neurol. 2018;25(10):1201-17. https://doi. org/10.1111/ene.13728

2. Huang P, He XY. Relationship between Alzheimer's disease and serum vitamin $\mathrm{D}$ and blood calcium levels. Theory and Practice of Medicine. 2017;30(18):2670-2. https://doi.org/10.19381/j. issn.1001-7585.2017.18.004

3. Cai JH, He Y, Zhong XL, Lei H, Wang F, Luo GH, et al. Magnetic resonance texture analysis in Alzheimer's disease. Acad Radiol. 2020;27(12):1774-83. https://doi.org/10.1016/j. acra.2020.01.006

4. Wei JP, Dai Y, Wen YQ. Discussing the pathogenesis of Alzheimer's disease from the theory of "yang qi, yin forming". J Trad Chin Med. 2018;59(24):2099-102. https://doi.org/10.13288/j.112166/r.2018.24.007

5. Greig SL. Memantine ER/Donepezil: a review in Alzheimer's disease. CNS Drugs. 2015;29(11):963-70. https://doi. org/10.1007/s40263-015-0287-2

6. Tian J, Sun RX, Wang P. Research progress in the pathogenesis of Alzheimer's disease. Shandong Med J [Internet]. 2018;58(27):97100. Available from: https://kns.cnki.net/kcms/detail/detail. aspx?FileName=SDYY201827027\&DbName=CJFQ2018

7. Wei R, Xie LZ. Progress in pharmacology and clinical application of Dengzhanxixin in treating cerebrovascular diseases. J Liaoning Univ Trad Chin Med. 2008;24(5):60-2. https://doi. org/10.13194/j.jlunivtcm.2008.05.62.weir.076

8. Lu JR, Tuo XP, Yu F. Effects of Schisandrone in Central China on learning and memory of Alzheimer's disease model rats and its mechanism. Shanxi Med J [Internet]. 2007;18(1):27-9. Available from: https://kns.cnki.net/kcms/detail/detail. aspx?FileName $=$ SXYY200701012\&DbName $=$ CJFQ2007

9. Benilova I, Karran E, De Strooper B. The toxic $A \beta$ oligomer and Alzheimer's disease: an emperor in need of clothes. Nat Neurosci. 2012;15(3):349-57. https://doi.org/10.1038/nn.3028

10. Sultana R, Perluigi M, Butterfield DA. Oxidatively modified proteins in Alzheimer's disease (AD), mild cognitive impairment and animal models of AD: role of Abeta in pathogenesis. Acta Neuropathol. 2009;118(1):131-50. https://doi.org/10.1007/ s00401-009-0517-0

11. Sankaranarayanan $S$, Price EA, Wu G, Crouthamel MC, Shi $X P$, Tugusheva $K$, et al. In vivo beta-secretase 1 inhibition leads to brain Abeta lowering and increased alpha-secretase processing of amyloid precursor protein without effect on neuregulin-1. J Pharmacol Exp Ther. 2008;324(3):957-69. https://doi.org/10.1124/jpet.107.130039

12. McConlogue L, Buttini M, Anderson JP, Brigham EF, Chen KS, Freedman SB, et al. Partial reduction of BACE1 has dramatic effects on Alzheimer plaque and synaptic pathology in APP Transgenic Mice. J Biol Chem. 2007;282(36):26326-34. https:// doi.org/10.1074/jbc.M611687200

13. Vintém AP, Henriques AG, da Cruz e Silva OA, da Cruz e Silva EF. PP1 inhibition by Abeta peptide as a potential pathological mechanism in Alzheimer's disease. Neurotoxicol Teratol. 2009;31(2):85-8. https://doi.org/10.1016/j.ntt.2008.11.001

14. Sinem F, Dildar K, Gökhan E, Melda B, Orhan Y, Filiz M. The serum protein and lipid oxidation marker levels in Alzheimer's disease and effects of cholinesterase inhibitors and antipsychotic drugs therapy. Curr Alzheimer Res. 2010;7(5):463-9. https:// doi.org/10.1016/10.2174/156720510791383822

15. Chen M, Jiang P, Lu J. Relationship between asymmetric dimethylarginine content and oxidative stress and the pathogenesis of Alzheimer's disease. Acta Pharm Sin. 2010;45(8):1001-5. https://doi.org/10.164 38/j.0513-4870.2010.08.009

16. Østergaard L, Aamand R, Gutiérrez-Jiménez E, Ho YC, Blicher $J U$, Madsen SM, et al. The capillary dysfunction hypothesis of Alzheimer's disease. Neurobiol Aging. 2013;(34):1018-31. https://doi.org/10.1016/j.neurobiolaging.2012.09.011

17. Jin K, Zhu Y, Sun Y, Mao XO, Xie L, Greenberg DA. Vascular endothelial growth factor (VEGF) stimulates neurogenesis in vitro and in vivo. Proc Natl Acad Sci U S A. 2002;99(18):1194650. https://doi.org/10.1073/pnas.182296499

18. Zhu X, Smith MA, Honda K, Aliev G, Moreira PI, Nunomura $A$, et al. Vascular oxidative stress in Alzheimer disease. J Neurol Sci. 2007;257(1-2):240-6. https://doi.org/10.1016/j. jns.2007.01.039 